Boise State University

ScholarWorks

Physics Faculty Publications and Presentations

Department of Physics

10-1-2009

\title{
Structural Development in Ge-Rich Ge-S Glasses
}

Y. Sakaguchi

Boise State University

Dmitri Tenne

Boise State University

Maria Mitkova

Boise State University

\section{(c) $\oplus \Theta \Theta$}

This is an author-produced, peer-reviewed version of this article. (C) 2009, Elsevier. Licensed under the Creative Commons AttributionNonCommercial-NoDerivatives 4.0 International License (https://creativecommons.org/licenses/by-nc-nd/4.0/). The final, definitive version of this document can be found online at Journal of Non-Crystalline Solids, doi: 10.1016/j.jnoncrysol.2009.04.064 
This is an author-produced, peer-reviewed version of this article. The final, definitive version of this document can be found online at Journal of Non-Crystalline Solids, published by Elsevier.

Copyright restrictions may apply. doi: 10.1016/j.jnoncrysol.2009.04.064

\title{
Structural Development in Ge-rich Ge-S glasses
}

\author{
Y. Sakaguchi ${ }^{1}$, D. A. Tenne ${ }^{2}$ and M. Mitkova ${ }^{1 *}$ \\ ${ }^{1}$ Department of Electrical and Computer Engineering, Boise State University, Boise, Idaho, 83725-2075, \\ ${ }^{2}$ Department of Physics, Boise State University, Boise, Idaho, 83725-1570
}

\begin{abstract}
The Raman spectra of Ge-S glasses in the Ge-rich region from Ge 33 to $46 \%$ have been investigated in order to know the structural development of the network glasses. From the detailed curve fits, we have found that there is an unassigned peak at $410 \mathrm{~cm}^{-1}$ and it becomes larger with increasing Ge composition. To clarify the structural origin of the peak, we virtually constructed the atomic arrangement of the glassy state starting from the crystalline state through the liquid state and changed the composition gradually depleting the medium in sulfur. From the consideration of the structural modeling and the atomic orbital theory, we suggest that single Ge-S chain is a probable structural origin of the peak.
\end{abstract}

PACS numbers: 61.05.Qr, 64.75Yz, 68.35bj

*Corresponding author. Tel. 1208426 3395; Fax 1208426 2470; e-mail:mariamitkova@ boisestate.edu (M. Mitkova)

\section{INTRODUCTION}

Ge-chalcogenide (Ge- $X: X=\mathrm{S}, \mathrm{Se})$ glasses are known as typical network glasses and are of a great interest because of their application potential. To utilize their attractive properties, it is quite important to know the exact structure of the materials. In the past few decades there has been an extensive effort to investigate the structure of the glassy Gechalcogenides. Results of several measurements have shown that a structural transition occurs with increasing Ge composition $^{1,2}$. According to the results of Mössbauer spectroscopy by Boolchand et al. ${ }^{2}$, at high Ge concentration there are three structural phases, $A, B$ and $C$, in these glasses. $A$ phase has similar structure to the high temperature crystalline phase of $\mathrm{GeX}_{2}$, which consists of tetrahedral $\mathrm{Ge}\left(X_{1 / 2}\right)_{4}$ units. $B$ phase consists of the ethane-like $(X$ $\left.{ }_{1 / 2}\right)_{3} \mathrm{Ge}-\mathrm{Ge}\left(X_{1 / 2}\right)_{3}$ units. $C$ phase has a double layer structure like crystalline(c-) $\mathrm{Ge} X$. The relative presence of these phases changes with increasing Ge composition. In recent years, the interest was mainly focused on the region around the critical composition of the rigidity percolation threshold, where the number of the constraints per atom is equal to the degree of freedom. In the composition region, a steep first-order-like transition, from very flexible floppy phase to rigid phase, is observed. The composition in Ge-Se system is Ge $23 \%$ [3]. In addition to that, in very recent years great attention has been paid to a narrow region near the critical composition, the "intermediate phase window", , where specific changes due to a self-organization occur [3, 4]. For Ge-Se system, the region is between Ge 20 and $26 \%$ [3].On the other hand, in the Ge-rich region from Ge 33 to $43 \%$, a much qualitative change is observed in the Raman spectra [1]. Indeed, it can be related to the structural transformations from $A$ to $B$ and from $B$ to $C$ phase. However, it is still worth examining the details of the structural development in the composition region because a quantitative analysis of Raman spectra has not been fully made yet. In this paper, we present the results of the detailed analysis of the Raman spectra of Ge-S glasses in the Ge-rich region, including our experimental data of thin films. On the basis of the results, we propose a unified model, which also explains other previous experimental results. Using the concept of the model, we finally provide perspective on the possibilities for applications. 
This is an author-produced, peer-reviewed version of this article. The final, definitive version of this document can be found online at Journal of Non-Crystalline Solids, published by Elsevier.

Copyright restrictions may apply. doi: 10.1016/j.jnoncrysol.2009.04.064

\section{EXPERIMENTAL}

Thin films of amorphous $\mathrm{Ge}_{46} \mathrm{~S}_{54}$ with the thickness of $300 \mathrm{~nm}$ were prepared by sputtering.

The composition was measured by the electron probe micro-analyzer in the system of the scanning electron microscope (LEO 1430VP). Raman spectra were recorded using a Raman spectroscopic system of Horiba Jobin Yvon T64000 equipped with liquid-nitrogen-cooled multichannel coupled-charge-device detector, in backscattering geometry. The power? ? $441.6 \mathrm{~nm} \mathrm{He}-\mathrm{Cd}$ laser line was used for excitation.

\section{RESULTS}

Fig.1 shows the Raman spectra of Ge-rich Ge-S glasses from Ge 33 to 46\%. The three Raman spectra, (a)-(c), are from the results of Takebe et al. [5], which were measured for bulk samples. The spectrum at Ge $46 \%$ was obtained using thin film prepared by sputtering in the present study. The qualitative change in the spectra is consistent with the results of Lucovsky et al. [1] and Kotsalas and Raptis [6]. A background component has been subtracted for the spectra at Ge 40 and $46 \%$. We performed curve fits with Gaussians to the spectra, assuming the presence of the peaks, which have already been assigned. Among the peaks, the peak at $340 \mathrm{~cm}^{-1}$ is attributed to the symmetric breathing mode of $\mathrm{S}$ atoms at $\mathrm{Ge}\left(\mathrm{S}_{1 / 2}\right)_{4}$ tetrahedron [7]. The intensity of the peak decreases with increasing Ge composition. This suggests that the number of the tetrahedral unit decreases with increasing Ge composition. At Ge $36 \%$, the content of $A$ phase is $0 \%$ [2]. Therefore, the peak at $340 \mathrm{~cm}^{-1}$ in the spectrum at Ge $36 \%$ should have other structural origin. According to Lucovsky [8], there is a vibrational frequency at $340 \mathrm{~cm}^{-1}$ of the ethane-like units, whose Raman active frequencies are at 240,340 and $376 \mathrm{~cm}^{-1}$. The presence of the ethane-like units indicates the presence of Ge-Ge bonds, in other words, chemical disorder in the system. The peak at $370 \mathrm{~cm}^{-1}$ is interpreted as the stretching motion of the outrigger raft (OR) accompanied by S-S bond, which was suggested by Bridenbaugh et al. [9], or the vibration of $S$ atoms on the edge-sharing double bonds [10, 11]. The vibrational frequency of the ethane-like units is also located near $370 \mathrm{~cm}^{-1}$ [8]. Jackson et al. point out from first-principles molecular-dynamics simulations that there are two peaks near $370 \mathrm{~cm}^{-1}$; a peak at $373 \mathrm{~cm}^{-1}$, the mode of the edge-sharing cluster, and a peak at $366 \mathrm{~cm}^{-1}$, the mode of the ethane-like cluster [12]. Overall, the peak at $370 \mathrm{~cm}^{-1}$ contains two components. One arises from ethane-like units. The other arises from and the edge-sharing double bonds or the stretching motion of OR. The peak at $430 \mathrm{~cm}^{-1}$ is regarded as the stretching mode of dimerized $\mathrm{S}$ atoms on the edge of the OR $[9,13]$. This is also a peak indicative of chemical disorder at a stoichiometric composition of Ge 33\%. The peak decreases with increasing Ge composition from 33 to $36 \%$, and almost vanishes at Ge 40\%. One can expect that the chance for creation of S-S bond declines with decreasing S composition and the result is consistent with the expectation. In 200-300 $\mathrm{cm}^{-1}$, we obtained two peaks located at 220 and $255 \mathrm{~cm}^{-1}$ from the curve fit. One of the vibrational frequencies of the ethane-like unit is located near $255 \mathrm{~cm}^{-1} ; 240 \mathrm{~cm}^{-1}$ according to Lucovsky et al. [8] and $255 \mathrm{~cm}^{-1}$ according to Jackson et al. [12]. In the spectra of c-GeS, there are two intensive peaks at 212 and $238 \mathrm{~cm}^{-1}$, which are the modes of double layer structure [14]. The peak at $220 \mathrm{~cm}^{-1}$ is considered to originate from these peaks. So far we made curve fits only using the peaks which have already been assigned. However, in the Ge-rich region more than $35 \%$, we could not fit the spectra without considering a peak at $410 \mathrm{~cm}^{-1}$, which becomes larger with increasing Ge composition. It is obvious that the peak at $410 \mathrm{~cm}^{-1}$ is not formed due to a shift of the peak at $430 \mathrm{~cm}^{-1}$ because both peaks were required to fit the spectrum at Ge $36 \%$. It seems that the increase of the peak at $410 \mathrm{~cm}^{-1}$ is related to the increase of $C$ phase. But there is no peak with such high frequency in the spectra of c-GeS. We would expect that there exists a structural unit, which has a stronger bond than that in the double layer and gives such high frequency vibration. As far as we know, no one suggests such a structural unit.

\section{DISCUSSION}

\section{A. Structural model}

In order to find out the structural origin of the vibrational mode at $410 \mathrm{~cm}^{-1}$, we have performed a virtual structural modeling. Fig. 2 shows how amorphous structure is formed from c-GeS 2 . High temperature crystalline phase of $\mathrm{GeS}_{2}$ consists of the tetrahedral units. In the figure, there are two streams of Ge-S chains. The stream can also be regarded as the sequence of corner-sharing tetrahedral units. The edge-sharing tetrahedral units connect the two streams. When c-GeS $S_{2}$ is heated and molten $\mathrm{GeS}_{2}$ is obtained from the high temperature crystalline phase, some Ge$\mathrm{S}$ covalent bonds would break. If a bond break occurs as shown in the figure, two neighboring $\mathrm{S}$ atoms become free and there is a chance to form a new S-S bond. This corresponds to the S-S dimmer on the edge of the OR. After breaking of the Ge-S bonds, the other counterparts of Ge atoms become also free. These atoms can bind each other 
This is an author-produced, peer-reviewed version of this article. The final, definitive version of this document can be found online at Journal of Non-Crystalline Solids, published by Elsevier.

Copyright restrictions may apply. doi: 10.1016/j.jnoncrysol.2009.04.064

and form a new Ge-Ge bond. As a result, the ethane-like unit is formed. Such structure, formed in the liquid phase is supposed to be preserved in the amorphous phase by quenching. This picture provides an answer to the fundamental question, why the chemical disorder exists in the stoichiometric composition and in amorphous phase.

To clarify the structure in Ge-rich Ge-S glasses, we subtracted $\mathrm{S}$ atoms from the structure of amorphous (a-) GeS $\mathrm{S}_{2}$ as shown in Fig.3. Here we subtracted $\mathrm{S}$ atoms out of the Ge-S chain and we assumed that the Ge-S chain structure is preserved. $\mathrm{S}$ atoms are classified into three groups; $1^{\text {st }}, 2^{\text {nd }}$ and $3^{\text {rd }}$ column, as shown in the figure. The $\mathrm{S}$ atoms in the $1^{\text {st }}$ column are the compositional elements of the Ge-S chain. The $\mathrm{S}$ atoms in the $2^{\text {nd }}$ column are at the edges of the corner-sharing tetrahedral units on the Ge-S chain, but not in the Ge-S chain. The $\mathrm{S}$ atoms in the $3^{\text {rd }}$ column are in the middle of the two Ge-S chains, and are out of the $2^{\text {nd }}$ column. By subtracting $\mathrm{S}$ atoms, the ethane-like unit will lose one $\mathrm{S}$ atom and $\mathrm{Ge}$ atom will seek other $\mathrm{S}$ atom. If there is a neighboring $\mathrm{S}$ atom in the $2^{\text {nd }}$ column, the Ge atom will bond with this $S$ atom. If there is no neighboring $S$ atoms in the $2^{\text {nd }}$ column, the Ge atom will bond with a $S$ atom in the $1^{\text {st }}$ column. Through such dynamics, we would expect that the interaction between the two streams of Ge-S chain becomes stronger, resulting in approaching of the two streams. Here, the ethane-like units play a role of glue. In fact, the composition variation of density indicates that there is considerable volume contraction in the region from Ge 33 to $45 \%$ [5, 15]. The same tendency is also observed in Ge-Se system [16]. We suggest that the large volume contraction in the region is caused by such an increase of the interaction between the Ge-S(Se) chains.

\section{B. Formation of layer structure}

To know further about the structural nature of the Ge-S glasses close to Ge $50 \%$, the diffraction study is useful. Using the results of the neutron diffraction study for Ge-Se system by Salmon's group [17, 18], we obtained that the bond angles of Se-Ge-Se are $111.01^{\circ}$ in a-GeSe 2 , and $101.57^{\circ}$ in liquid (1-) $\mathrm{GeSe}_{2}$, which are close to $109.47^{\circ}$, while the bond angle is $94.82^{\circ}$ in $1-\mathrm{GeSe}$, which is close to $90^{\circ}$. Recent $a b$ initio molecular-dynamics simulations by Van Roon et al. support these bond angles [19]. The change in the bond angle from Ge 33 to $50 \%$ can be explained in terms of the atomic orbital theory. The outermost electronic configuration of $\mathrm{Ge}$ is $(4 s)^{2}(4 p)^{2}$ and that of Se is $(4 s)^{2}(4 p)^{4}$. In Se atom, two $s$ electrons are low in energy and they do not participate in bonding. Two electrons among four $p$ electrons are used to form covalent bond with other two atoms. Hence, Se atom makes twofold coordination. The remaining two $p$ electrons do not participate in bonding and form a pair called "lone-pair electrons". For Ge atom, there are two cases depending on the composition. When the Se composition is large enough, a tetrahedral structure will be formed centering a Ge atom. In this case, $s p^{3}$ hybridization occurs in the Ge atom by the promotion of one $s$ electron to $p$ level. The formation of four $s p^{3}$ orbitals results in the four equivalent covalent bonds. Thus, the bond angle is close to $109.47^{\circ}$, which is the value for a perfect tetrahedron. When the Ge composition becomes $50 \%$, twofold coordinated Ge-Se chains are formed, as depicted in Fig. 4(a), as a result of the subtraction of Se atoms. In order to form twofold coordination in Ge atom, two $p$ electrons would participate in bonding, leaving $s$ electrons in bonding without forming hybridization. Since two $p$ orbitals have normal orientation to each other, the bond angle of Se-Ge-Se is fixed to be $90^{\circ}$. In Fig.4(b), we illustrate how a double layer structure can be formed from the Ge-Se chains. The Ge-Se chains are alternatively aligned along $b$-axis at different height in $c$ axis. They are connected to each other having a third bond. This is the way how a layer structure is formed from the chains and the layer becomes 'double'. The formation of the third bond can also be explained in terms of the atomic orbital theory. In Se atom, two $p$ electrons are used for covalent bonds and two $p$ electrons remain as lone-pair electrons. In Ge atom, two $p$ electrons are used for covalent bonds and one $p$ orbital is empty. As it is well known in the formation of ammonium ion $\mathrm{NH}_{4}{ }^{+}$, a coordinate bond is formed between a lone-pair orbital and an empty orbital. Applying this concept to this situation, we can expect that a new coordinate bond could be formed between a Ge atom and a Se atom, which are in different neighboring chains.

\section{Origin of the peak at $410 \mathrm{~cm}^{-1}$}

The important point in the third bond formation is that the bond formation is possible only for hetero-polar pair. According to the neutron diffraction study, there exist homo-polar bonds in 1-GeSe [18]. The homo-polar bonds should only exist in the Ge-Se chain composed of covalent bonds. This leads to the situation that some atoms in the chain face homo-polar atoms in a neighboring chain. At the portion, the atoms do not bond with the faced atoms and the chains can fail to form double layer. In the region, the Ge-Se chain is left as a single chain without being involved in the layer. The structure in the liquid might be quenched in amorphous phase. Although we dealt with Ge-Se system in the above discussion, the situation could be the same in Ge-S system since the atomic organization and chemical bonding are very similar. Therefore, such single chain in Ge-S system would be a possible structural origin of the peak at $410 \mathrm{~cm}^{-1}$. 


\section{CONCLUSION}

We have made an analysis of Raman spectra of Ge-rich Ge-S glasses and found that there is an unassigned peak, which is located at $410 \mathrm{~cm}^{-1}$, in the region from Ge 36 to $46 \%$. In order to know the structural origin of the peak, we considered the structural development in the atomic arrangement starting from the crystal structure of $\mathrm{GeS}_{2}$ and proposed a structural model of Ge-rich Ge-S glasses. The model explains why the ethane-like units appear in the glasses. The interaction between the two Ge-S chains becomes stronger with increasing Ge composition due to the role of the ethane-like units. This is consistent with the data of the density measurements. We have also demonstrated that a new type of bond, which bridges the two Ge-S chains to form double layer structure, is supposed to appear in Ge-rich region close to $50 \%$ using a concept of atomic orbital theory. From these our considerations, we have speculated that there is incomplete portion in the double layer structure in Ge-rich Ge-S glasses close to $50 \%$ and proposed the structural origin of the Raman peak at $410 \mathrm{~cm}^{-1}$ as single Ge-S chain. Although we could not give direct evidence in the present study, such possibility will be examined by firstprinciples molecular-dynamics simulations. Our model emphasizes the uniqueness of amorphous structure. The concept of our model can provide a useful guideline to find a new functionality in Ge-S glasses in Ge-rich region. The unique nature in the interaction between Ge-S chains can affect optoelectronic response and photodiffusion of silver. In addition, the low dimensionality in the structure may be utilized in producing a new type of nanostructures. It would be interesting to examine these possibilities in further studies.

\section{ACKNOWLEDGMENTS}

The authors are grateful to Professor. C. Raptis and Professor K. Nakamura for invaluable discussion and to Professor H. Takebe and Professor P. Boolchand for permission to use their data for analysis. The authors also thank Idaho Microfabrication Laboratory in Boise State University for technical assistance in composition measurements. Y.S. acknowledges support from IMI- NFG (NSF Grant No. DMR-0409588 and DMR-049588).

\section{REFERENCES:}

${ }^{1}$ G. Lucovsky, F. L. Galeener, R. C. Keezer, R. H. Geils and H. A. Six, Phys. Rev. B 10, 5134 (1974).

2 P. Boolchand, J. Grothaus, M. Tenhover, M. A. Hazle and R. K. Grasselli, Phys. Rev. B 33, 5421 (1986).

[3] P. Boolchand, X. Feng and W. J. Bresser, J. Non-Cryst. Solids 293-295, 358 (2001).; P. Boolchand, D. G. Georgiev and B. Goodman, J. Optoelectron. Adv. Mater. 3, 703 (2001).

[4] A. Sarbaeva, S. A. Wells, A. Huerta and M. F. Thorpe, Phys. Rev. B 75, 224204 (2007).

[5] H. Takebe, H. Maeda and K. Morinaga, J. Non-Cryst. Solids 291, 14 (2001).

[6] I. P. Kotsalas and C. Raptis, Phys. Rev. B 64, 125210 (2001).

[7] G. Lucovsky, J. P. deNeufville and F. L. Galeener, Phys. Rev. B 9, 1591 (1974).

[8] G. Lucovsky, R. J. Nemanich and F. L. Galeener, in Proceedings of the $7^{\text {th }}$ International Conference on Amorphous and Liquid Semiconductors, Edinburgh, Scotland, 1977, edited by W. E. Spear (G. G. Stevenson, Dundee, Scotland, 1977), p.125. 
[9] P. M. Bridenbaugh, G. P. Espinosa, J. E. Griffiths, J. C. Phillips and J. P. Remeika, Phys. Rev. B 20, 4140 (1979).

[10] S. Sugai, Phys. Rev. B 35, 1345 (1987).

[11] K. Murase, K. Inoue and O. Matsuda, in: Y. Sakurai, Y. Hamakawa, T. Masumoto, K. Shirae and K. Suzuki (Eds.), Current Topics in Amorphous Materials: Science and Technology, Elesevier, Amsterdam, 1993, p.47.

[12] K. Jackson, A. Briley, S. Grossman, D. V. Porezag and M. R. Pederson, Phys. Rev. B 60, R14985 (1999).

[13] M. Yamaguchi, T. Shibata and K. Tanaka, J. Non-Cryst. Solids 234, 715 (1998).

[14] J. D. Wiley, W. J. Buckel and R. L. Schmidt, Phys. Rev. B 13, 2489 (1976).

[15] Y. Kawamoto and S. Tsuchihashi, J. Am. Ceram. Soc. 54, 131 (1971).

[16] A. Feltz, H. Aust and A. Blayer, J. Non-Cryst. Solids 55, 179 (1983).

[17] P. S. Salmon and I. Petri, J. Phys.: Condens. Matter 15, S1509 (2003).

[18] I. Petri, P. S. Salmon and H. E. Fischer, J. Phys.: Condens. Matter 11, 7051 (1999).

[19] F. H. M. van Roon, C. Massobrio, E. de Wolff and S. W. de Leeuw, J. Chem. Phys. 113, 5425 (2000). 


\section{Figure captures}

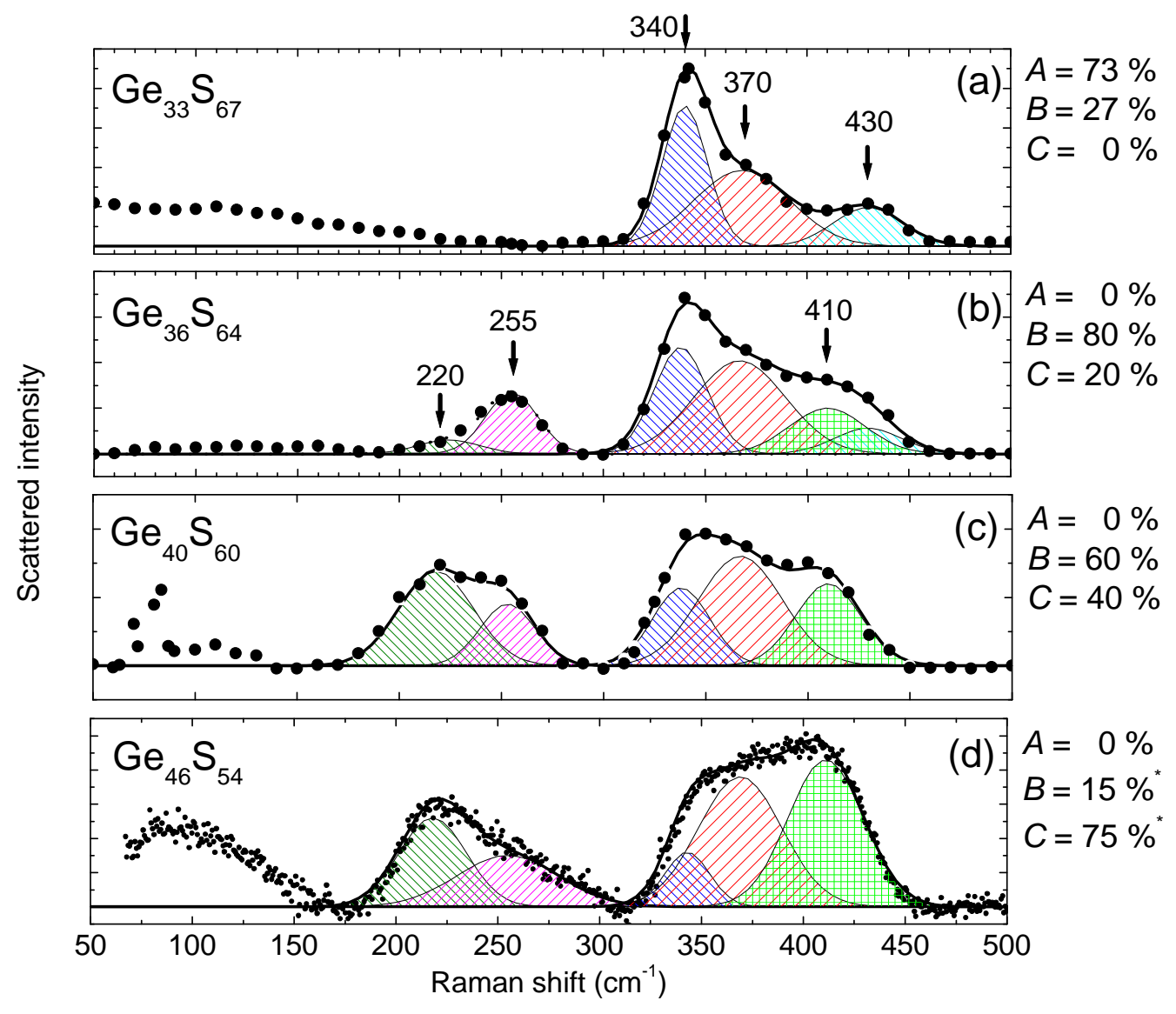

Fig. 1 Raman spectra of Ge-S glasses and the results of the curve fit. The dots show the experimental Raman spectra. The solid curves show the results of fitting, which are the sum of the indicated peaks. The contents of $A, B$ and $C$ phase, which were obtained from Mössbauer spectroscopy [2], are also indicated. The values at Ge $46 \%\left(^{*}\right)$ were estimated from the exraporation of the results. 


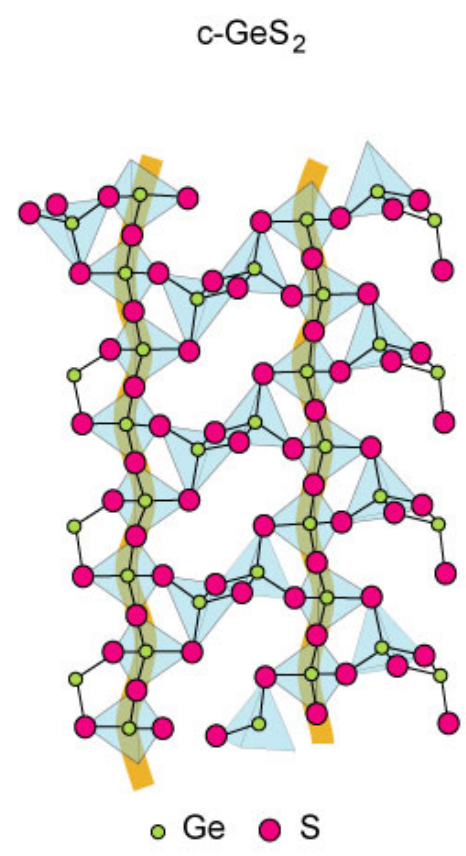

$\mathrm{I}-\mathrm{GeS}_{2}$

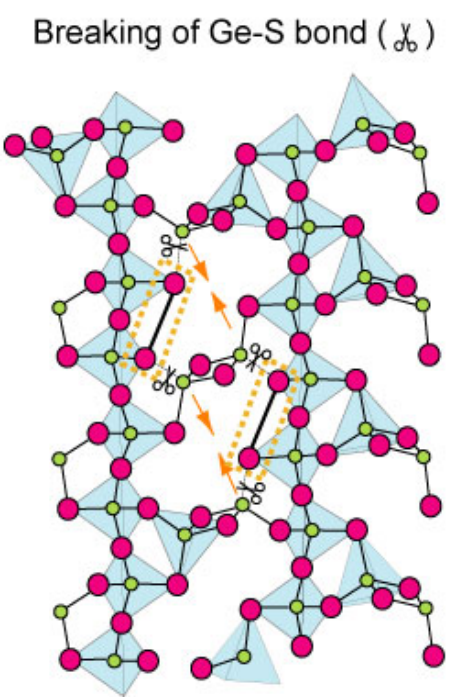

S-S dimer on the edge of the OR
$\mathrm{a}-\mathrm{GeS}_{2}$

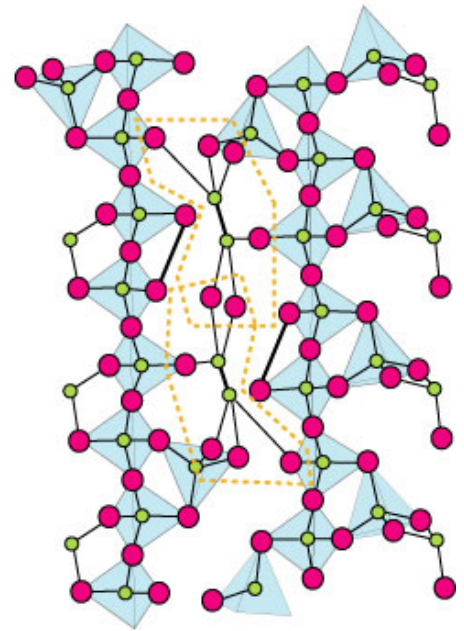

ethane-like unit

Fig. 2 A structural model, which explains how chemical disorder is formed in amorphous $\mathrm{GeS}_{2}$ from crystalline $\mathrm{GeS}_{2}$ through the liquid phase. 


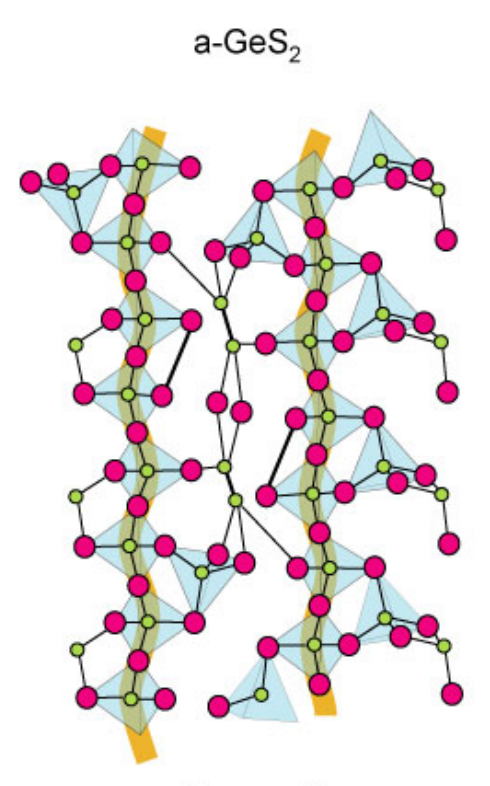

$\circ \mathrm{Ge} \circ \mathrm{S}$

\section{Subtracting $S$ atoms}

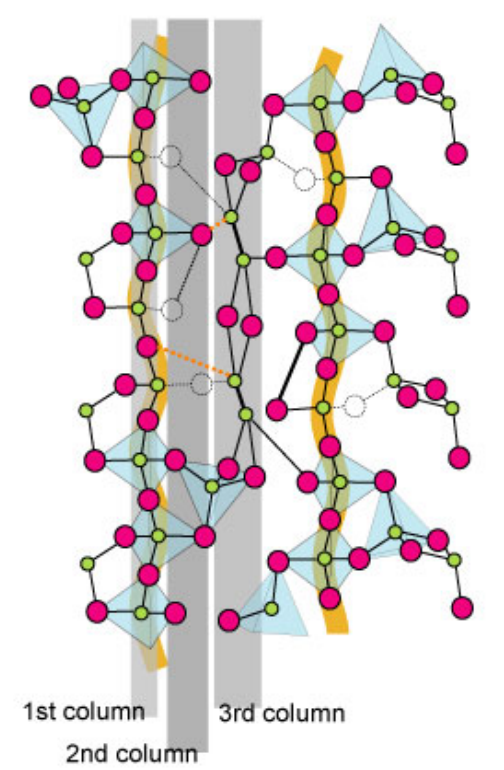

Much more subtracting $\mathrm{S}$ atoms

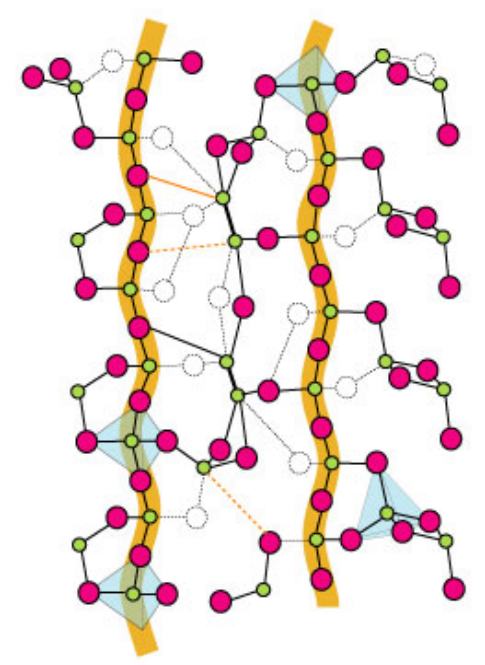

Fig. 3 A model of the structural development in Ge-S glasses from Ge $33 \%\left(\mathrm{a}-\mathrm{GeS}_{2}\right)$ to Ge-rich region. 
after subtracting Se atoms to be Ge $50 \%$

Formation of double layer (b)

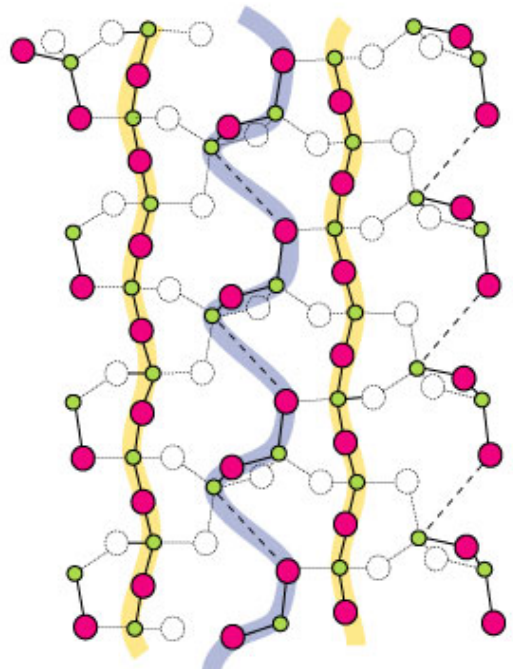

(a)

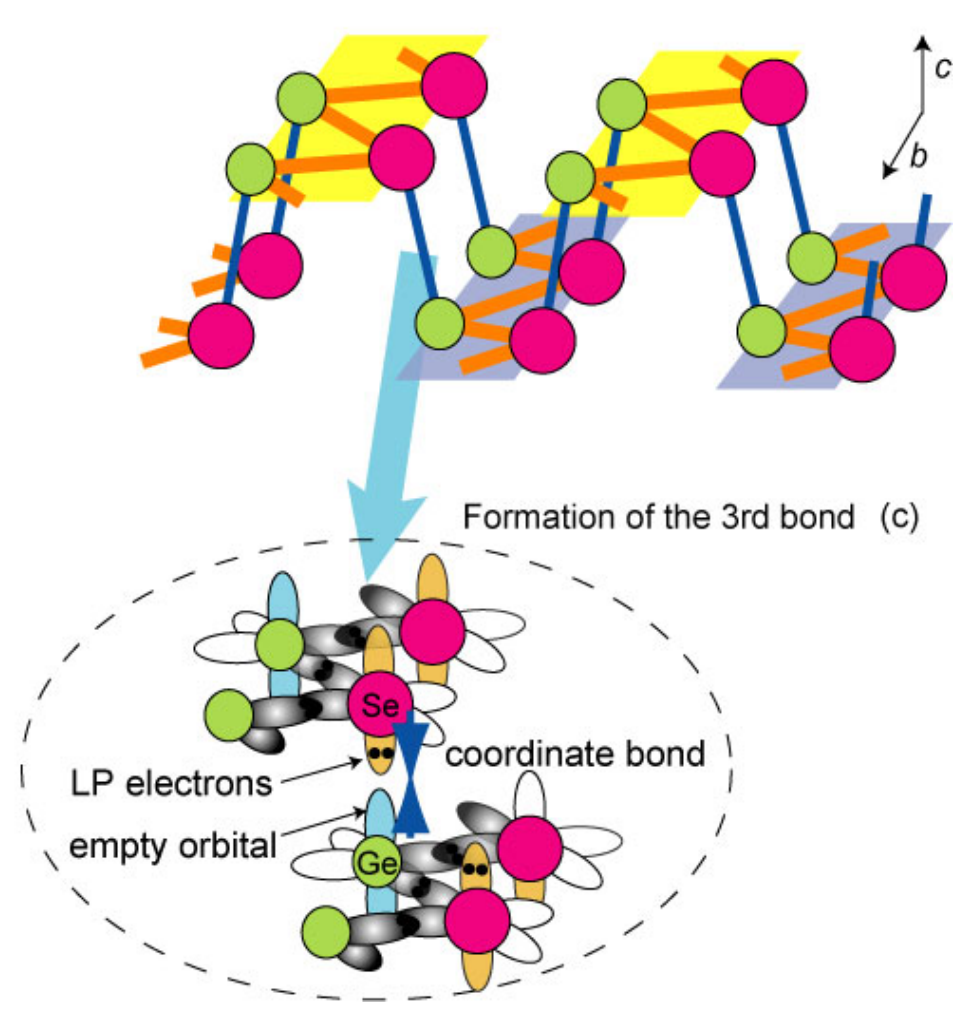

Fig. 4 (a) Atomic arrangement at Ge $50 \%$. Se atoms are subtracted from the structure of $\mathrm{GeSe}_{2}$. (b) Formation of the double layer structure from Ge-Se chains. Color of the chain corresponds to that in (a). (c) Formation of the third bond (indicated by blue in (b)). 